\title{
Retraction Note: Glycol chitosan incorporated retinoic acid chlorochalcone (RACC) nanoparticles in the treatment of Osteosarcoma
}

\author{
Yan-Guo Qin ${ }^{*}$, Lan-Yu Zhu², Chen-Yu Wang ${ }^{3}$, Bo-Yan Zhang ${ }^{3}$, Qing-Yu Wang ${ }^{1}$, Rui-Yan Li ${ }^{1}$ and Zhen Liu ${ }^{1}$
}

\author{
Retraction to: Lipids Health Dis 14, 70 (2015) \\ https://doi.org/10.1186/s12944-015-0068-4
}

The Editor in Chief has retracted this article [1] because of significant concerns regarding a number of Figures presented in this work, which question the integrity of the data. The authors have contacted the journal to say they were unable to replicate data presented in Figure $2 \mathrm{~A}$ and $\mathrm{C}$. In addition, concerns were raised by readers regarding:

- Figure $3 \mathrm{~A}$ and $3 \mathrm{~B}$ - similarities between bands within western blot

- Figure 4A- several areas appear to be repeated throughout the panels. It also appears Figure 4A was published by unrelated authors in a different article, which has since been retracted [2]

- Figure 6 - it appears the graphs representing results from different cell lines are very similar, but with different labels and quantification.

- Figure 7D - similarities between bands within each blot. It appears some bands have been repeated. It also appears a similar Western blot has been published earlier [3] in a different article by unrelated authors as Figure 4A.
- Figure 8 C, D, E and $\mathrm{F}$ where it appears several areas of the image are repeated throughout that image.

The authors did not respond to these concerns. They stated that the results cannot be reproduced.

The corresponding author Yan-Guo Qin stated on behalf of all co-authors that they agree to this retraction.

\section{Author details \\ 'Department of Orthopedics, The Second Hospital of Jilin University, Changchun 130041, Jilin, China. ${ }^{2}$ Nursing School, Changchun University of Chinese Medicine, Changchun 130117, Jilin, China. ${ }^{3}$ Norman Bethune Medical School, Jilin University, Changchun 130021, Jilin, China.}

Published online: 30 November 2020

References

1. Qin Y, Zhu L, Wang C, Zhang B, Wang Q, Li R, Liu Z. Glycol chitosan incorporated retinoic acid chlorochalcone (RACC) nanoparticles in the treatment of Osteosarcoma. Lipids Health Dis. 2015. https://doi.org/10.1186/ s12944-015-0068-4.

2. RETRACTED Lu H, Ma J, Zhuang Z, Zhang Y, Cheng H, Shi J. Retinoic acidincorporated glycol chitosan nanoparticles inhibit the expression of Ezh2 in U118 and U138 human glioma cells Retraction in /10.3892/mmr.2016.5130. Mol Med Rep. 2015. https://doi.org/10.3892/mmr.2015.4294.

3. Zhong J, Hou J. A novel retinoic acid chalcone reverses epithelialmesenchymal transition in prostate cancer cells. Bangladesh J Pharmacol. 2015. https://doi.org/10.3329/bjp.v10i2.22602. 